\title{
Poster Abstract: Equalized Recovery: Weakening Invariance for Control and Estimation
}

\author{
Kwesi Rutledge \\ Ann Arbor, Michigan \\ krutledg@umich.edu
}

\author{
Sze Zheng Yong \\ Tempe, Arizona \\ szyong@asu.edu
}

\author{
Necmiye Ozay \\ Ann Arbor, Michigan \\ necmiye@umich.edu
}

\begin{abstract}
When deployed into real environments, control systems need to be able to operate when their sensor data can become 'missing' (e.g., a vehicle's radar system may incorrectly detect a falling leaf as a vehicle on the road, or a distributed control system may lose sensor data packets while attempting to transmit). Guaranteeing safety of such systems can be handled by enforcing boundedness of the state or the estimated state of a system during operation. The form of boundedness that we use within this work is called equalized recovery and the goal of this work is to find controllers or estimators that satisfy equalized recovery in the presence of missing data. Equalized recovery relaxes the notion of invariance and allows the system states to be in a larger set during missing data events as long as the states can be steered back to the original set. Prefix-based controllers and estimators are introduced to solve this problem and methods to synthesize them are presented.
\end{abstract}

\section{CCS CONCEPTS}

- Theory of computation $\rightarrow$ Timed and hybrid models; Logic and verification; $\bullet$ Computing methodologies $\rightarrow$ Computational control theory;

\section{KEYWORDS}

Robust estimators, bounded-error estimation, missing data, invariance control

\section{ACM Reference Format:}

Kwesi Rutledge, Sze Zheng Yong, and Necmiye Ozay. 2019. Poster Abstract: Equalized Recovery: Weakening Invariance for Control and Estimation. In Proceedings of 22nd ACM International Conference on Hybrid Systems: Computation and Control (HSCC '19). ACM, New York, NY, USA, 2 pages. https://doi.org/10.1145/3302504.3313358

\section{INTRODUCTION}

The problems of sensor occlusion and communication packet drops are inescapable for autonomous systems operating in the real world. For example, an autonomous vehicle radar system is impacted by weather or environmental conditions that are (at least currently) out of a system designer's control. This provides the foundational question: How does one design an autonomous system that can operate safely when 'missing data' events can occur?

Permission to make digital or hard copies of part or all of this work for personal or classroom use is granted without fee provided that copies are not made or distributed for profit or commercial advantage and that copies bear this notice and the full citation on the first page. Copyrights for third-party components of this work must be honored.

For all other uses, contact the owner/author(s).

HSCC '19, April 16-18, 2019, Montreal, QC, Canada

(C) 2019 Copyright held by the owner/author(s)

ACM ISBN 978-1-4503-6282-5/19/04.

https://doi.org/10.1145/3302504.3313358

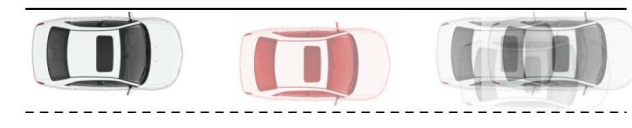

Figure 1: An adaptive cruise controller is a safety-critical component of an autonomous vehicle that must be tolerant to missing data events. Although the radar signal (or other sensors) may lose measurements due to weather or road geometry, the system should still be able to avoid crashes.

Designing control and estimation systems that are robust to missing data events has been discussed in several contexts. In [6], the notion of missing data in a control system is expressed in a probabilistic setting where all probabilities are conditioned on an information set that may increase (if a new measurement is received) or remain constant (if measurement is missing) in size over time. In [8], the Kalman Filter's equations are analyzed and restructured to show how distributions and update rules should change when missing data events occur.

While the previous methods were based on disturbances as random variables of unbounded, infinite support and making guarantees about the behavior of the controlled system as time goes to infinity, this work's disturbances or uncertainties belong to bounded sets without notions of a distribution and we aim to make guarantees about finite-time behavior of the controlled system. The inclusion of these assumptions aligns this work with the fields of $\ell_{\infty}$ estimation [2] and set-valued estimation (first introduced by [1] and discussed more deeply in [7]).

\section{ESTIMATOR AND CONTROLLER SYNTHESIS}

To express one possible notion of formal guarantees and safety, this work considers a finite-time boundedness property that we call equalized recovery [3]. A discrete-time dynamical system with state $x(t)$ is said to achieve an equalized recovery level $M_{1}$ with recovery time $T$ and intermediate level $M_{2} \geq M_{1}$ at time $t_{0}$ if whenever $\left\|x\left(t_{0}\right)\right\| \leq M_{1}$, we have $\|x(t)\| \leq M_{2}$ for all $t \in\left[t_{0}, t_{0}+T\right]$ and $\left\|x\left(t_{0}+T\right)\right\| \leq M_{1}$.

This property is especially useful when performing control or estimation on dynamical systems that experience missing data events, as the conclusion of bounded state estimation error can be made by identifying this relaxed condition instead of equalized performance, which requires a uniform bound at all times. For the remainder of this section, we will discuss the estimation problem to save space, but these results also apply to the control problem.

The estimation problem statement can be described as follows: With an uncertain initial state belonging to a set described by parameter $M_{1}$, a known dynamical system $\Sigma$, missing data language 


$$
\begin{aligned}
& x(k+1)= A(k) x(k)+B(k) u(k)+w(k), \quad w(k) \in \mathcal{W}, \\
& y(k)=\left\{\begin{array}{ll}
C x(k)+v(k), & q(k)=1, \\
\emptyset, & q(k)=0,
\end{array} \quad v(k) \in \mathcal{V}\right. \\
& \mathcal{L}=
\end{aligned}
$$

Figure 2: An example system specification where the affine dynamics are given with all matrices known. The evolution of the discrete state $q(k)$ is restricted to one of the sequences outlined in $\mathcal{L}$. Each row of the language $\mathcal{L}$ represents a word (i.e. a possible sequence of $q(k)$ ), where the circles can be interpreted as: a filled circle indicates that data is available in that time step and an empty circle indicates that data is 'missing.'

$\mathcal{L}$ and known process and measurement disturbance sets $\mathcal{W}, \mathcal{V}$, find a set of estimator gains that guarantees the satisfaction of the equalized recovery property for recovery level $M_{1}$, intermediate level $M_{2}$ and recovery time $T$. As further explanation, consider a model as specified in Fig. 2. In this specification, $\mathcal{L}$ contains all possible sequences of $q(k)$, sequences of 1 and 0 . Each sequence is represented by a row of circles in $\mathcal{L}$, where the filled and unfilled circles indicate whether data is available or missing, respectively.

We then propose a mixture of time-based [3] and prefix-based [4] estimator designs that can be found using robust linear programs. While an estimator based on state augmentation exists as well [5], we do not discuss it here to focus our comparison's scope.

\section{EXAMPLES}

We will use examples from the automotive domain to demonstrate these ideas. The first application considered is an adaptive cruise control system where the goal is to keep a safe distance from the vehicle in front by adjusting the ego vehicle's speed (see Fig. 1). While driving, faults in the measurement of headway (e.g. from the radar not detecting the cars in front of you during a turn) can lead to 'missing data' for some instances of time. In our example, we consider data events where all car sensor data become missing in a pattern that can be summarized by the rule: ' 1 measurement in a specific time window is missing.' Solutions are generated in the form of time-based or prefix-based estimators and the two types of estimators are compared based on the minimum intermediate level $M_{2}$ that each can guarantee.

To demonstrate the control design problem with missing data, a simple lane keeping system is analyzed. This simple system attempts to remain invariantly in a neighborhood of the lane center during missing data events. Fig. 3 shows how our prefix-based controller behaves when identical initial conditions and adversarial disturbances are chosen by the environment. In this graph, different traces are created by different missing data patterns occurring (all other variables mentioned in the previous sentence remain the same across traces). For all missing data patterns, under the boundary

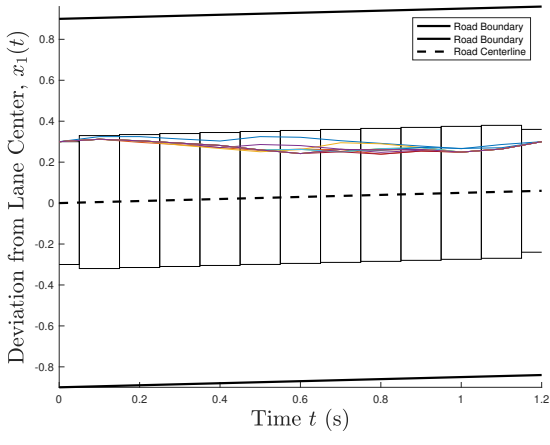

Figure 3: The car's lane keeping controller is evaluated under various missing data patterns when the system experiences hard initial conditions and adversarial disturbances. Here, the system starts on the edge of the hyperbox defined by $M_{1}$ and experiences an identical sequence of maximum norm disturbances during each test (each trace). The missing data pattern is different for each trace shown. This demonstrates that for any missing data pattern in $\mathcal{L}$ the controlled system remains within our guaranteed bounds.

initial condition and adversarial disturbances, the system does not violate the designed bounds.

Current work focuses on the connection between equalized recovery, tube-based model predictive control and controlled invariant sets, as well as the extension of our work to replace the hyperboxes in the equalized recovery condition with more general set descriptions.

\section{ACKNOWLEDGMENTS}

This work was made possible by an Early Career Faculty grant from NASA's Space Technology Research Grants Program, grant N0001418-1-2501 from the Office of Naval Research, NSF grant ECCS1553873, and NSF Graduate Research Fellowship Grant Number DGE 1256260.

\section{REFERENCES}

[1] D. Bertsekas and I. Rhodes. 1971. Recursive state estimation for a set-membership description of uncertainty. IEEE Trans. Automat. Control 16, 2 (1971), 117-128.

[2] M. Milanese and A. Vicino. 1991. Optimal estimation theory for dynamic systems with set membership uncertainty: an overview. Automatica 27, 6 (1991), 9971009.

[3] K. J. Rutledge, S. Z. Yong, and N. Ozay. 2018. Optimization-Based Design of Bounded-Error Estimators Robust to Missing Data. IFAC-PapersOnLine ADHS Full Papers (July 2018). Analysis and Design of Hybrid Systems ADHS.

[4] K. J. Rutledge, S. Z. Yong, and N. Ozay. 2019 (Accepted). Prefix-based Boundederror Estimation with Intermittent Observations. 2019 American Control Conference (ACC) (2019 (Accepted)).

[5] Q. Shen S. M. Hassaan and S. Z. Yong. 2019 (Accepted). Bounded-Error Estimator Design with Missing Data Patterns via State Augmentation. 2019 American Control Conference (ACC) (2019 (Accepted)).

[6] L. Schenato, B. Sinopoli, M. Franceschetti, K. Poolla, and S. S. Sastry. 2007. Foundations of Control and Estimation Over Lossy Networks. Proc. IEEE 95, 1 (Jan 2007), 163-187. https://doi.org/10.1109/JPROC.2006.887306

[7] J. S. Shamma and K.-Y. Tu. 1999. Set-valued observers and optimal disturbance rejection. IEEE Trans. on Automatic Control 44, 2 (1999), 253-264.

[8] B. Sinopoli, L. Schenato, M. Franceschetti, K. Poolla, M. I. Jordan, and S. S. Sastry. 2004. Kalman filtering with intermittent observations. IEEE Trans. Automat. Control 49, 9 (Sep. 2004), 1453-1464. https://doi.org/10.1109/TAC.2004.834121 